Int. J. Contemp. Math. Sciences, Vol. 2, 2007, no. 4, 195 - 201

\title{
The Outputs Estimation and Improvement of Efficiency on Interval Data in DEA
}

\author{
M. Navabakhsh \\ Dept. of Sociality Science and Research Branches \\ Islamic Azad University, Tehran, Iran \\ F. Hosseinzadeh Lotfi, T. Allahviranloo ${ }^{1}$, F. Rezai Balf \\ Department of Mathematics, Science and Research Branch \\ Islamic Azad University,Tehran, Iran ${ }^{2}$
}

\section{H. Zhiani Rezai}

Department of Mathematics, Islamic Azad University

Mashhad, Iran

\begin{abstract}
This paper develops the presented method by Yan et al. [Eur. J. Operat. Res. 136 (2002) 19]. In this paper, the methods are introduced for evaluating the outputs of Decision Making Units (DMUs) by using interval data in Data Envelopment Analysis (DEA), when some or all inputs in an interval decision making unit (IDMU), fully are increased.

Mathematics Subject Classification: Operations Research, No.90.
\end{abstract}

Keywords: Data Envelopment Analysis, Inverse Data Envelopment Analysis model, Multi-objective programming.

\section{INTRODUCTION}

We know that data envelopment analysis (DEA) models can be used to estimate output levels of a decision making unit (DMU) when some or all inputs are increased, so that the efficiency level of the under-evaluated unit preserved

\footnotetext{
${ }^{1}$ Corresponding author, Email addresses: alahviranlo@yahoo.com

${ }^{2}$ Tel.:+98-21-44804172, Fax: +98-21-44804172, P.O. Box 14155/775 and 14155/4933, Post code: 1477893855
} 
or increased to some extent, so efficiency level of the other DMUs maintain without varies. In this paper we generalize this idea for interval data. This paper is organized as follows: In section 2 the DEA technique is presented. In section 3 the estimate of the outputs with interval data is presented as well. In section 4 an example is solved and finally conclusions are drowning in section 5.

\section{BACKGROUND}

Consider $n$ units, each using $m$ inputs produce $s$ outputs. Also assume that $X_{p}=\left(x_{1 p}, \ldots, x_{m p}\right)$ and $Y_{p}=\left(y_{1 p}, \ldots, y_{s p}\right)$ are inputs and outputs vectors $D M U_{p}$ respectively, where, $X_{p} \geq 0, X_{p} \neq 0, Y_{p} \geq 0, Y_{p} \neq 0$. The efficiency $D M U_{p}$ in the output-oriented CCR model is obtained as follows:

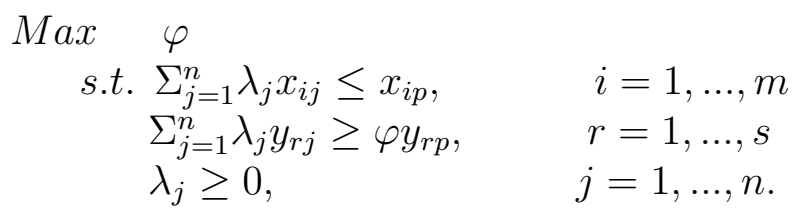

The dual problem of model (1) is expressed in the following model, with components of the vectors and as variables.

$$
\begin{array}{rlr}
\text { Min } & \sum_{i=1}^{m} v_{i} x_{i p} \\
\text { s.t. } & \sum_{r=1}^{s=1} u_{r} y_{r p}=1, \\
& \sum_{i=1}^{m} v_{i} x_{i j}-\sum_{r=1}^{s} u_{r} y_{r j} \geq 0, \quad j=1, \ldots, n \\
& u_{r}, v_{i} \geq 0, &
\end{array}
$$

Assume that in model (2) the levels of inputs and outputs are known to lie within bounded intervals, i.e. $x_{i j} \in\left[x_{i j}^{L}, x_{i j}^{U}\right]$ and $y_{r j} \in\left[y_{r j}^{L}, y_{r j}^{U}\right]$, with the upper and lower bounds of intervals given as constant and assumed strictly positive. Then, model (2) will be non-linear from the original variables $u_{1}, u_{2}, \ldots, u_{s}$ and $v_{1}, v_{2}, \ldots, v_{m}$, also the level of $x_{i j}$ inputs and $y_{r j}$ outputs are variables which exact values are to be estimated. In such case, for linearizing model (2), we apply the following transformation for the variables $x_{i j}$ and $y_{r j}$ :

$\begin{array}{ll}x_{i j}=x_{i j}^{L}+s_{i j}\left(x_{i j}^{U}-x_{i j}^{L}\right), & 0 \leq s_{i j} \leq 1, \quad i=1, \ldots, m, j=1, \ldots, n \\ y_{r j}=y_{r j}^{L}+t_{r j}\left(y_{r j}^{U}-y_{r j}^{L}\right), & 0 \leq t_{r j} \leq 1, \quad r=1, \ldots, s, j=1, \ldots, n\end{array}$

With the above transformations, model (2) is converted into the following linear program:

$\operatorname{Min} \quad \sum_{i=1}^{m} v_{i}\left(x_{i p}^{L}+s_{i p}\left(x_{i p}^{U}-x_{i p}^{L}\right)\right)$ 
s.t. $\sum_{r=1}^{s} u_{r}\left(y_{r p}^{L}+t_{r p}\left(y_{r p}^{U}-y_{r p}^{L}\right)\right)=1$, $1, \ldots, n$

$$
\begin{aligned}
& \sum_{i=1}^{m} v_{i}\left(x_{i j}^{L}+s_{i j}\left(x_{i j}^{U}-x_{i j}^{L}\right)\right)-\sum_{r=1}^{s} u_{r}\left(y_{r j}^{L}+t_{r j}\left(y_{r j}^{U}-y_{r j}^{L}\right)\right) \geq 0, \\
& u_{r}, v_{i} \geq 0, \quad i=1, \ldots, m, r=1, \ldots, s .
\end{aligned}
$$

In which, $0 \leq s_{i j} \leq 1, i=1, \ldots, m, j=1, \ldots, n$ and $0 \leq t_{r j} \leq 1, r=$ $1, \ldots, s, j=1, \ldots, n$

Now, with introducing the new variables $q_{i j}=v_{i} s_{i j}$ and $p_{r j}=u_{r} t_{r j}$, in which the variables $q_{i j}$ and $p_{r j}$ satisfy the condition $0 \leq q_{i j} \leq v_{i}$ and $0 \leq p_{r j} \leq u_{r}$, respectively. The model (3) is converted as follows:

$$
\begin{aligned}
& \left.\operatorname{Min} \quad \sum_{i=1}^{m} v_{i} x_{i p}^{L}+q_{i p}\left(x_{i p}^{U}-x_{i p}^{L}\right)\right) \\
& \text { s.t. } \left.\sum_{r=1}^{s} u_{r} y_{r p}^{L}+p_{r p}\left(y_{r p}^{U}-y_{r p}^{L}\right)\right)=1 \text {, } \\
& \left.\left.\sum_{i=1}^{m} v_{i} x_{i j}^{L}+q_{i j}\left(x_{i j}^{U}-x_{i j}^{L}\right)\right)-\sum_{r=1}^{s} u_{r} y_{r j}^{L}+p_{r j}\left(y_{r j}^{U}-y_{r j}^{L}\right)\right) \geq 0, \\
& 1, \ldots, n \\
& p_{r j} \leq u_{r}, \quad r=1, \ldots, s, j=1, \ldots, n \\
& q_{i j} \leq v_{i}, \quad i=1, \ldots, m, j=1, \ldots, n \\
& p_{r j} \geq 0, q_{i j} \geq 0, \quad r=1, \ldots, s, i=1, \ldots, m, j= \\
& 1, \ldots, n \\
& v_{i}, u_{r} \geq 0, \quad i=1, \ldots, m, r=1, \ldots, s .
\end{aligned}
$$

The CCR DEA model with exact input-output data derives as a special case of model (4). Indeed, if the lower and upper bounds coincide for all inputs and outputs, the bounded intervals are all of zero length. Therefore, the second terms in the summations vanish together with the variables $p_{r j}$ and $q_{i j}$, the constraints of the form $p_{r j} \leq u_{r}$ and $q_{i j} \leq v_{i}$ are eliminated and model (4) is converted into model (2). In model below, two models (5) and (6), provide lower bound and upper bound of the efficiency scores for DMU, respectively $[1]$.

$$
\begin{array}{rlr}
\text { Min } & z_{p}^{L}=\sum_{i=1}^{m} v_{i} x_{i p}^{U} \\
\text { s.t. } & \sum_{r=1}^{s} u_{r} y_{r p}^{L}=1, \\
& \sum_{i=1}^{m} v_{i} x_{i p}^{U}-\sum_{r=1}^{s} u_{r} y_{r p}^{L} \geq 0, & \\
& \sum_{i=1}^{m} v_{i} x_{i j}^{L}-\sum_{r=1}^{s} u_{r} y_{r j}^{U} \geq 0, & \\
& u_{r}, v_{i} \geq 0, &
\end{array}
$$

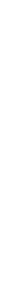




$$
\begin{array}{ll}
\sum_{i=1}^{m} v_{i} x_{i j}^{U}-\sum_{r=1}^{s} u_{r} y_{r j}^{L} \geq 0, & j=1, \ldots, n, j \neq p \\
u_{r}, v_{i} \geq 0, & i=1, \ldots, m, r=1, \ldots, s .
\end{array}
$$

The dual model (5) and (6) are given as follows, respectively.

$$
\begin{aligned}
& \operatorname{Max} \varphi^{L}
\end{aligned}
$$

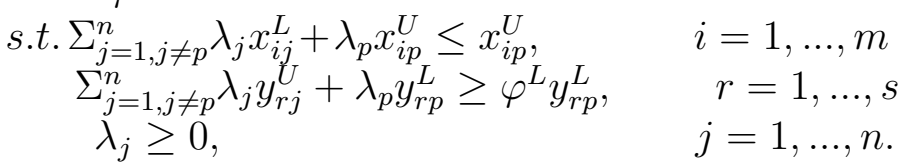

and

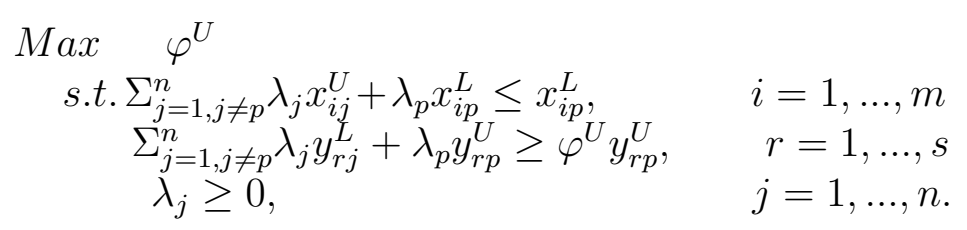

Therefore, the models $(7)$ and $(8)$, provide a bounded interval $\left[\varphi^{L *}, \varphi^{U *}\right]$ for each unit, in which its efficiency scales lie from the worst to the best cases.

\section{The ESTIMATE OF THE OUTPUTS WITH IN- TERVAL DATA}

Recently, a few papers have been published which discuss the follow question: we increase certain inputs if among a group of DMUs, to a particular unit and the efficiency level of the other DMUs maintain without changes, how much the output levels as well as improvement of the objective function can be increased? To amswer this question, some methods have been proposed by Wei et al. [2], Yan et al. [3], Jahanshahloo et al. [4]. Here, we develop their ideas for interval data, using two models (7) and (8). Suppose that $\beta_{p}=\left(\beta_{1}, \beta_{2}, \ldots, \beta_{s}\right)$ are estimating output levels, on the condition that $\varphi^{L *}$ and $\varphi^{U *}$ are improved $\left(1-\frac{\gamma}{100}\right) \varphi^{L *}$ and $\left(1-\frac{\mu}{100}\right) \varphi^{U *}$, when inputs $x_{p}^{L}$ and $x_{p}^{U}$ are increased to $\alpha_{p}^{L}$ and $\alpha_{p}^{U}$, in this two models (7) and (8), in which both of the models are shown as follows, respectively.

$$
\begin{array}{cc}
\text { Max } \quad \beta_{p}=\left(\beta_{1}, \beta_{2}, \ldots, \beta_{s}\right) & \\
\text { s.t. } \sum_{j=1, j \neq p}^{n} \lambda_{j} x_{i j}^{L}+\lambda_{p} x_{i p}^{U} \leq \alpha_{i p}^{U}, & i=1, \ldots, m \\
\sum_{j=1, j \neq p}^{n} \lambda_{j} y_{r j}^{U}+\lambda_{p} y_{r p}^{L} \geq\left(\left(1-\frac{\gamma}{100}\right) \varphi^{L *}\right) \beta_{r p}, & r=1, \ldots, s \\
\beta_{r p} \geq y_{r p}^{L}, & r=1, \ldots, s \\
\lambda_{j} \geq 0, & j=1, \ldots, n .
\end{array}
$$

In which $\alpha_{p}^{U}=x_{p}^{U}+\Delta x_{p}^{U}$, so that, $\Delta x_{p}^{U} \geq 0$ and 


$$
\begin{array}{cc}
\text { Max } \quad \beta_{p}=\left(\beta_{1}, \beta_{2}, \ldots, \beta_{s}\right) & \\
\text { s.t. } \sum_{j=1, j \neq p}^{n} \lambda_{j} x_{i j}^{U}+\lambda_{p} x_{i p}^{L} \leq \alpha_{i p}^{L}, & i=1, \ldots, m \\
\sum_{j=1, j \neq p}^{n} \lambda_{j} y_{r j}^{L}+\lambda_{p} y_{r p}^{U} \geq\left(\left(1-\frac{\gamma}{100}\right) \varphi^{U *}\right) \beta_{r p}, & r=1, \ldots, s \\
\beta_{r p} \geq y_{r p}^{U}, & r=1, \ldots, s \\
\lambda_{j} \geq 0, & j=1, \ldots, n .
\end{array}
$$

In which $\alpha_{p}^{L}=x_{p}^{L}+\Delta x_{p}^{L}$, so that, $\Delta x_{p}^{L} \geq 0$. Notice that always, $\alpha_{p}^{L} \leq \alpha_{p}^{U}$.

To solve multi-objective programming problems (9) and (10), we use weighted sum single-objective programming models, with the objective function $\frac{1}{s} \sum_{r=1}^{s} w_{r} \beta_{r p}$. In which, $w_{r}$ s the weights are determined by the manager or any other users.

\section{NUMERICAL EXAMPLE}

To illustrate the above, consider the interval data of Table 1 ( 5 units with 2 inputs and 2 outputs) which are given together with the efficiency scales obtained by applying models (7) and (8).

Table 1. Interval Data, Efficiency Scores

\begin{tabular}{|c|c|c|c|c|c|c|}
\hline DMU & Input I & Input II & output I & output II & Lower Efficiency & Upper Efficiency \\
\hline $\mathrm{j}$ & $x_{1 j}$ & $x_{2 j}$ & $y_{1 j}$ & $y_{2 j}$ & $\varphi^{U *}$ & $\varphi^{L *}$ \\
\hline \hline 1 & {$[12,15]$} & {$[0.21,0.48]$} & {$[138,144]$} & {$[21,22]$} & 4.464 & 1 \\
\hline 2 & {$[10,17]$} & {$[0.1,0.7]$} & {$[143,159]$} & {$[28,35]$} & 4.405 & 1 \\
\hline 3 & {$[4,12]$} & {$[0.16,0.35]$} & {$[157,198]$} & {$[21,29]$} & 1.215 & 1 \\
\hline 4 & {$[19,22]$} & {$[0.12,0.19]$} & {$[158,181]$} & {$[21,25]$} & 2.247 & 1.103 \\
\hline 5 & {$[14,15]$} & {$[0.06,0.09]$} & {$[157,161]$} & {$[28,40]$} & 1 & 1 \\
\hline
\end{tabular}

In order to illustrate Table 1, consider $D M U_{3}$, as a sample among five DMUs. Hence, with respect to Table $1, D M U_{3}$ has the optimal values $\varphi^{L *}=1$ and $\varphi^{U *}=1.215$, which both of them are the efficiency scales of $D M U_{3}$ obtained by applying models (7) and (8), respectively. Assume that, $\alpha_{3}^{U}=x_{3}^{U}+\Delta x_{3}^{U}=$ $[12+2,0.35+0.05]$ and $\alpha_{3}^{L}=x_{3}^{L}+\Delta x_{3}^{L}=[4+1,0.16+0.02]$. We would like, the efficiency improvement of $D M U_{3}$ to be up to $\% 25 \varphi^{L *}(\gamma=25)$, and $\% 10 \varphi^{U *}(\mu=10)$, respectively. In order to determine the output DMU level, from models (9) and (10), we solve the following multi-objective programming problems, respectively:

$$
\begin{array}{cl}
\operatorname{Max} & \beta=\left(\beta_{1}, \beta_{2}\right) \\
\text { S.t. } & 12 \lambda_{1}+10 \lambda_{2}+12 \lambda_{3}+19 \lambda_{4}+14 \lambda_{5} \leq 14 \\
& 0.21 \lambda_{1}+0.1 \lambda_{2}+0.35 \lambda_{3}+0.12 \lambda_{4}+0.06 \lambda_{5} \leq 0.4 \\
& 144 \lambda_{1}+159 \lambda_{2}+157 \lambda_{3}+181 \lambda_{4}+161 \lambda_{5}-0.75 \beta_{1} \geq 0 \\
& 22 \lambda_{1}+35 \lambda_{2}+21 \lambda_{3}+25 \lambda_{4}+40 \lambda_{5}-0.9 \beta_{2} \geq 0 \\
& \beta_{1} \geq 157 \\
& \beta_{2} \geq 21
\end{array}
$$




$$
\lambda_{1}, \lambda_{2}, \lambda_{3}, \lambda_{4}, \lambda_{5} \geq 0
$$

The following weighted sum model solves the problem above:

$$
\begin{aligned}
\text { Max } & \beta=2 \beta_{1}+3 \beta_{2} \\
\text { S.t. } & 12 \lambda_{1}+10 \lambda_{2}+12 \lambda_{3}+19 \lambda_{4}+14 \lambda_{5} \leq 14 \\
& 0.21 \lambda_{1}+0.1 \lambda_{2}+0.35 \lambda_{3}+0.12 \lambda_{4}+0.06 \lambda_{5} \leq 0.4 \\
& 144 \lambda_{1}+159 \lambda_{2}+157 \lambda_{3}+181 \lambda_{4}+161 \lambda_{5}-0.75 \beta_{1} \geq 0 \\
& 22 \lambda_{1}+35 \lambda_{2}+21 \lambda_{3}+25 \lambda_{4}+40 \lambda_{5}-0.9 \beta_{2} \geq 0 \\
& \beta_{1} \geq 157 \\
& \beta_{2} \geq 21 \\
& \lambda_{1}, \lambda_{2}, \lambda_{3}, \lambda_{4}, \lambda_{5} \geq 0
\end{aligned}
$$

In which, the normal vector $(2,3)$ in objective function is the same weight vector, which is determined at manager or any other users discretion.

The optimal solution and the objective optimal value are as follow:

$\left(\lambda_{1}, \lambda_{2}, \lambda_{3}, \lambda_{4}, \lambda_{5}, \beta_{1}, \beta_{2}\right)=(0,1.4,0,0,0,296.8,54.4)$ and $\beta^{*}=757$ and

$$
\begin{aligned}
\operatorname{Max} & \beta=\left(\beta_{1}, \beta_{2}\right) \\
\text { S.t. } & 15 \lambda_{1}+17 \lambda_{2}+4 \lambda_{3}+22 \lambda_{4}+15 \lambda_{5} \leq 5 \\
& 0.48 \lambda_{1}+0.7 \lambda_{2}+0.16 \lambda_{3}+0.19 \lambda_{4}+0.09 \lambda_{5} \leq 0.18 \\
& 138 \lambda_{1}+143 \lambda_{2}+198 \lambda_{3}+158 \lambda_{4}+157 \lambda_{5}-0.91 \beta_{1} \geq 0 \\
& 21 \lambda_{1}+28 \lambda_{2}+29 \lambda_{3}+21 \lambda_{4}+28 \lambda_{5}-1.1 \beta_{2} \geq 0 \\
& \beta_{1} \geq 198 \\
& \beta_{2} \geq 29 \\
& \lambda_{1}, \lambda_{2}, \lambda_{3}, \lambda_{4}, \lambda_{5} \geq 0
\end{aligned}
$$

The following weighted sum model solves the above problem:

$$
\begin{aligned}
\text { Max } & \beta=2 \beta_{1}+3 \beta_{2} \\
\text { S.t. } & 15 \lambda_{1}+17 \lambda_{2}+4 \lambda_{3}+22 \lambda_{4}+15 \lambda_{5} \leq 5 \\
& 0.48 \lambda_{1}+0.7 \lambda_{2}+0.16 \lambda_{3}+0.19 \lambda_{4}+0.09 \lambda_{5} \leq 0.18 \\
& 138 \lambda_{1}+143 \lambda_{2}+198 \lambda_{3}+158 \lambda_{4}+157 \lambda_{5}-0.91 \beta_{1} \geq 0 \\
& 21 \lambda_{1}+28 \lambda_{2}+29 \lambda_{3}+21 \lambda_{4}+28 \lambda_{5}-1.1 \beta_{2} \geq 0 \\
& \beta_{1} \geq 198 \\
& \beta_{2} \geq 29 \\
& \lambda_{1}, \lambda_{2}, \lambda_{3}, \lambda_{4}, \lambda_{5} \geq 0
\end{aligned}
$$

In which, the normal vector $(2,3)$ in objective function is the same weight vector, which is determined by manager.

The optimal solution and the objective optimal value are as follow:

$\left(\lambda_{1}, \lambda_{2}, \lambda_{3}, \lambda_{4}, \lambda_{5}, \beta_{1}, \beta_{2}\right)=(0,0,1.1,0,0.04,246.8,30.1)$ and $\beta^{*}=583.7$

\section{CONCLUSION}

In this paper, using data envelopment analysis with interval data, we deter- 
mine how to produce the output levels when some or all inputs of an interval decision making unit (IDMU) are increased, such as efficiency scale is improved. For each DMU, we consider two problems, so that, they are solved using multi-objective programming problem. To solve this problem, we use the single-objective programming problem. Finally, we mentioned, in general, the increase of the outputs is not unique, because, the multi-objective problems usually have the multiple solutions.

\section{REFERENCES}

[1] D. K. Despotis, Y. G. Smirlis, Data envelopment analysis with imprecise data, European Journal of Operational Research 140, 24-36, 2002.

[2] Q.L. Wei, J. Zhang, X. Zhang, An inverse DEA model for input/output estimate, European Journal of Operational Research 121 (1) 151-163, (2000). [3] H. Yan, Q.L. Wei, G. Hao, DEA models for resource reallocation and production input/output estimation, European Journal of Operational Research $136,1931,2002$.

[4] D. K. Despotis, Y. G. Smirlis, Data envelopment analysis with imprecise data, European Journal of Operational Research 140, 24-36, 2002.

[5] G. R. Jahanshahloo, F. Hosseinzadeh Lotfi, N. Shoja, G. Tohidi and S. Razavyan, The outputs estimation of a DMU according to improvement of its efficiency, Applied Mathematics and Computation, 147 Issue 2, 12 January, Pages 409-413, 2004.

\section{Received: October 7, 2005}

